\title{
ASYMPTOTIC EXPANSION OF THE STOKES SOLUTIONS AT SMALL VISCOSITY: THE CASE OF NON-COMPATIBLE INITIAL DATA*
}

\author{
GUNG-MIN GIE ${ }^{\dagger}$
}

\begin{abstract}
Without imposing the so-called compatibility condition on the initial data, we obtain an asymptotic expansion of the Stokes solutions at small viscosity $\varepsilon$ as the sum of the linearized Euler solution and a corrector function, which balances the discrepancy on the boundary of the Stokes and the linearized Euler solutions. Using such an expansion and smallness of the corrector, as the viscosity $\varepsilon$ tends to zero, we obtain the uniform $L^{2}$ convergence of the Stokes solutions to the linearized Euler solution with rate of order $\varepsilon^{1 / 4}$.
\end{abstract}

Key words. Boundary layers, singular perturbations, Stokes equations, non-compatible initial data, curvilinear coordinate system.

AMS subject classifications. 35B25, 35C20, 35K05, 76D07, 76D10.

\section{Introduction}

The motion of an incompressible viscous fluid, in a smooth and bounded domain, is described by the Navier-Stokes equations, which are classically supplemented with the no-slip boundary condition, i.e., the fluid is at rest on the boundary. In fluid dynamics, one interesting open problem is to study the asymptotic behavior of the Navier-Stokes solution at small viscosity in a bounded domain. More precisely, considering the Euler equations (with the same initial data and external force as for the Navier-Stokes) as the inviscid limit of the Navier-Stokes equations, we are interested in the so-called vanishing viscosity limit problem to verify if the Navier-Stokes solution converges to the Euler solution as the viscosity tends to zero in, e.g., $L^{2}$ in space uniformly in time. Being first-order, the Euler equations need only the impermeable boundary condition, reflecting no entry or exit of fluid from the domain. Hence, due to the discrepancy between the Navier-Stokes and Euler solutions at the boundary, we expect the boundary layers to occur. Prandtl [23] was the first to make real progress on analyzing these boundary layer effects, and much of a pragmatic nature has been discovered. However, up to this point, the mathematical understanding is still very inadequate to answer the vanishing viscosity limit problem. Concerning the boundary layer analysis related to the Navier-Stokes equations, we refer the readers to, e.g., $[3,4,7,8,9,10,13,17,18,25,29,30]$.

In studying the viscous boundary layers, some simplified models of the NavierStokes equations can be made useful, in which the effects of nonlinearity and/or the divergence-free constraint are weakened. For instance, in $[15,16]$, asymptotic behavior of the radially symmetric flow in a unit disk is studied where the NavierStokes equations are reduced essentially to a $1 \mathrm{D}$ heat equation in the radial variable $r \in[0,1)$. For this simple model, one can prove that the vanishing viscosity limit holds true. Moreover, it is known that the difference of the viscous (Navier-Stokes) and

${ }^{*}$ Received: August 26, 2012; accepted (in revised form): March 23, 2013. Communicated by Francois Golse.

${ }^{\dagger}$ Department of Mathematics, University of California, Riverside, 900 University Ave., Riverside, CA 92521, USA (ggie@math.ucr.edu). 
inviscid (Euler) solutions near the boundary, i.e., at $r=1$, can be described by

$$
-2 V_{0}(r=1) \operatorname{erfc}\left(\frac{1-r}{\sqrt{2 \varepsilon t}}\right)-2 \int_{0}^{t} F(r=1, t=s) \operatorname{erfc}\left(\frac{1-r}{\sqrt{2 \varepsilon(t-s)}}\right) d s,
$$

where $V_{0}$ and $F$ are the initial data and external force of the viscous and inviscid problems, and $\operatorname{erfc}(\cdot)$ is the complimentary error function defined in (4.7) below. Hence we see that the boundary layer of the radially symmetric flow is caused in part by the ill-prepared initial data $V_{0}$, which does not vanish on the boundary, and by the external force $F$. (The boundary layer caused by an ill-prepared initial data can be understood physically as an impulsively started motion at $t=0$ near the boundary.) Furthermore, from (1.1), we see that the boundary layer caused by $V_{0}$ is more problematic to handle than that produced by $F$, because its derivatives in $r$ or $t$ are more singular in time than the derivatives of the boundary layer caused by $F$. In this sense, we notice that it is crucial to handle the case of ill-prepared initial data in the boundary layer analysis of viscous fluids. A similar type of analysis is valid for other symmetric flows as well, e.g., the so-called plane-parallel or infinite-pipe flow studied in, e.g., $[19,20,22]$ or $[12,21]$.

As another type of simplifications of the Navier-Stokes equations, by linearizing around the zero (or any divergence-free) tangential flow, we obtain the Stokes (or Oseen) equations. Boundary layers of the Stokes equations are well-studied in, e.g., $[5,11,27,28]$. As a problem closely related to the Stokes, but a bit more general, boundary layers of the Oseen type equations in a channel domain is studied in, e.g., [24]; see Remark 1.1 below. However, in the articles mentioned above, only the case of well-prepared initial data, which vanishes on the boundary, is considered. As explained in Section 3 of [24], to handle the case of ill-prepared initial data, one main difficulty is enforcing the divergence-free constraint on the corrector that satisfies the desired boundary condition. In [11] and [5], a divergence-free corrector is introduced while it causes a small error on the boundary. To manage this small error, the authors introduce the supplementary corrector as a solution of the stationary Stokes equations with a constant viscosity. But this approach requires the compatibility condition on the initial data.

In this article, as a continuation and generalization of earlier works mentioned above, we study the boundary layers of the Stokes equations for ill-prepared initial data; see (1.6) below. More precisely, we consider the Stokes equations in a bounded domain $\Omega$ in $\mathbb{R}^{3}$ with smooth boundary $\Gamma$,

$$
\begin{cases}\frac{\partial \boldsymbol{u}^{\varepsilon}}{\partial t}-\varepsilon \Delta \boldsymbol{u}^{\varepsilon}+\nabla p^{\varepsilon}=\boldsymbol{f}, & \text { in } \Omega \times(0, T), \\ \operatorname{div} \boldsymbol{u}^{\varepsilon}=0, & \text { in } \Omega \times(0, T), \\ \boldsymbol{u}^{\varepsilon}=0, & \text { on } \Gamma \times(0, T), \\ \left.\boldsymbol{u}^{\varepsilon}\right|_{t=0}=\boldsymbol{u}_{0}, & \text { in } \Omega,\end{cases}
$$

where $\varepsilon$ is small but strictly positive viscosity parameter, $T>0$ is a fixed time, and $\boldsymbol{f}$ and $\boldsymbol{u}_{0}$ are given smooth data satisfying (1.6).

We introduce the function spaces

$$
\begin{aligned}
& H=\left\{\boldsymbol{v} \in L^{2}(\Omega) \mid \operatorname{div} \boldsymbol{v}=0, \boldsymbol{v} \cdot \boldsymbol{n}=0 \text { on } \Gamma\right\}, \\
& V=\left\{\boldsymbol{v} \in H_{0}^{1}(\Omega) \mid \operatorname{div} \boldsymbol{v}=0\right\}
\end{aligned}
$$


and $V^{\prime}$ as the dual space of $V$. Here $\boldsymbol{n}$ is the outer unit normal vector on $\Gamma$.

The existence, uniqueness, and regularity results for solutions of (1.2) are classical (see, e.g., [26]): For $\boldsymbol{u}_{0} \in H$ and $\boldsymbol{f} \in L^{2}\left(0, T ; V^{\prime}\right)$ there exists a unique solution $\left(\boldsymbol{u}^{\varepsilon}, p^{\varepsilon}\right)$ of (1.2) such that

$$
\boldsymbol{u}^{\varepsilon} \in L^{2}(0, T ; V) \cap C([0, T] ; H), \quad \frac{\partial \boldsymbol{u}^{\varepsilon}}{\partial t} \in L^{2}\left(0, T ; V^{\prime}\right) .
$$

Formally setting $\varepsilon=0$ in (1.2), we obtain the limit problem

$$
\begin{cases}\frac{\partial \boldsymbol{u}^{0}}{\partial t}+\nabla p^{0}=\boldsymbol{f}, & \text { in } \Omega \times(0, T), \\ \operatorname{div} \boldsymbol{u}^{0}=0, & \text { in } \Omega \times(0, T), \\ \boldsymbol{u}^{0} \cdot \boldsymbol{n}=0, & \text { on } \Gamma \times(0, T), \\ \left.\boldsymbol{u}^{0}\right|_{t=0}=\boldsymbol{u}_{0}, & \text { in } \Omega .\end{cases}
$$

Our main task in this article is to construct a divergence-free corrector that balances the discrepancy on the boundary of the Stokes and linearized Euler solutions. Toward this end, as appearing in Section 4 below, we modify the method introduced in related works $[7,8,25]$. We believe that this method of constructing the corrector can be made useful in many boundary layer problems of incompressible flows. (See Section 6 as well for some remarks on the higher-order expansions of the Stokes solutions.)

REMARK 1.1. The Oseen type equations considered in [24] are the linearization of the Navier-Stokes equations around a stationary and tangential velocity $\boldsymbol{U}^{\infty}$. (When $\boldsymbol{U}^{\infty}$ is equal to zero, we obtain the Stokes equations.) Especially for a channel domain case, as shown in [24], one can essentially get rid of the terms involved in $\boldsymbol{U}^{\infty}$ by using the semi-Lagrangian coordinates on the time and tangential variables. Hence, we believe that our analysis in this article is applicable to the Oseen type equations in [24]. Boundary layers of the Oseen type equations in a 3D smooth domain with a general vector field $\boldsymbol{U}^{\infty}$ will be studied elsewhere as a future project.

From the boundary layer analysis point of view, to study the asymptotic expansion of $\boldsymbol{u}^{\varepsilon}$ with respect to small viscosity $\varepsilon$, a certain amount of regularity on the limit solution $\boldsymbol{u}^{0}$ to (1.5) is required. Hence, throughout this article, we assume that

$$
\boldsymbol{u}_{0} \in H \cap H^{4}(\Omega), \quad \boldsymbol{f} \in L^{\infty}\left(0, T ; H \cap H^{4}(\Omega)\right),
$$

so that the limit problem (1.5) is well-posed with the regularity results in Lemma 2.1 below. Here we do not assume that the tangential component of $\boldsymbol{u}_{0}$ vanishes on the boundary; i.e., the smooth initial data $\boldsymbol{u}_{0}$ is in $H$ only, but not necessarily in $V$.

For the analysis below, we assume that the domain $\Omega$, which may not be convex, is connected and sufficiently regular:

$$
\Gamma \text { is of class } C^{5} \text {. }
$$

However, since the boundary layer is a highly local phenomenon, all the analysis in this article can easily extend to treat more general domains in $\mathbb{R}^{3}$, enclosed by finitely many compact and simply connected boundaries which do not intersect with each other. 
The article is organized as follows: In Section 2, we prove the well-posedness of the linearized Euler equations (1.5) and then state the main result Theorem 2.7 on the convergence of the Stokes solutions to the linearized Euler solution as the viscosity tends to zero. To establish the main result, in Section 3, a curvilinear system adapted to the boundary is introduced to manage the geometrical difficulties of the problem. In Section 4, by solving the Prandtl equation associated with the Stokes equations, we construct a divergence-free corrector which allows us to obtain an asymptotic expansion of the Stokes solution as the sum of the linearized Euler solution and the corrector. Here, using the fact that the main part of the corrector is essentially a heat solution in a half plane, we also derive some estimates on the corrector. In Section 5, by performing the energy estimates on the difference of the Stokes solution and the proposed asymptotic expansion, and by using the estimates on the corrector, we prove Theorem 2.7. In addition, concerning well-prepared initial data in (6.1), in Section 6 we demonstrate how one can generalize the method of constructing the corrector in this article to obtain the higher-order expansions of the Stokes solution at small viscosity.

\section{Well-posedness of the limit problem and main result}

We first state and prove the well-posedness result of the linearized Euler equations (1.5):

Lemma 2.1. Under the assumption (1.6), for any time $T>0$ there exists a unique solution $\left(\boldsymbol{u}^{0}, p^{0}\right)$ ( $p^{0}$ is unique up to an additive constant) of (1.5) such that

$$
\boldsymbol{u}^{0} \in L^{\infty}\left(0, T ; H \cap H^{4}(\Omega)\right), \quad \frac{\partial \boldsymbol{u}^{0}}{\partial t} \in L^{\infty}\left(0, T ; H \cap H^{4}(\Omega)\right),
$$

and

$$
p^{0} \in L^{\infty}\left(0, T ; H^{5}(\Omega)\right)
$$

Proof. To determine the pressure $p^{0}$, from $(1.5)_{1,2}$, we infer that

$$
\Delta p^{0}=\operatorname{div} \boldsymbol{f}, \text { in } \Omega .
$$

Since $\boldsymbol{u}_{0}$ is in $H$, differentiating $(1.5)_{3}$ in $t$, we see that $\partial\left(\boldsymbol{u}^{0} \cdot \boldsymbol{n}\right) / \partial t=0$ on $\Gamma$. Hence, by multiplying $(1.5)_{1}$ by $\boldsymbol{n}$, we find

$$
\nabla p^{0} \cdot \boldsymbol{n}=\boldsymbol{f} \cdot \boldsymbol{n}, \text { on } \Gamma .
$$

Solving the Neumann problem (2.3) and (2.4) for $p^{0}$, we obtain (2.2).

Now, using the given pressure $p^{0}$, we rewrite $(1.5)_{1}$ in the form

$$
\frac{\partial \boldsymbol{u}^{0}}{\partial t}=\boldsymbol{f}-\nabla p^{0}
$$

and find

$$
\boldsymbol{u}^{0}=\boldsymbol{u}_{0}+\int_{0}^{t}\left(\boldsymbol{f}-\nabla p^{0}\right) d s
$$

hence (2.1) follows.

Since the uniqueness of the solution $\left(\boldsymbol{u}^{0}, p^{0}\right)$ is easy to verify, the proof of Lemma 2.1 is complete. 
REMARK 2.2. If $\boldsymbol{f} \equiv 0$ in (1.2) and (1.5), from the proof of Lemma 2.1 it is easy to see that the linearized Euler solution $\boldsymbol{u}^{0}$ is stationary:

$$
\boldsymbol{u}^{0}=\boldsymbol{u}_{0}
$$

In this case, if we additionally assume that $\boldsymbol{u}_{0} \in V$, then $\boldsymbol{u}^{0}=0$ on $\Gamma$. Hence, without introducing any corrector function, one can verify the convergence of $\boldsymbol{u}^{\varepsilon}$ to $\boldsymbol{u}^{0}$ in the sense that

$$
\left\|\boldsymbol{u}^{\varepsilon}-\boldsymbol{u}^{0}\right\|_{L^{\infty}\left(0, T ; L^{2}(\Omega)\right)} \leq \kappa_{T} \varepsilon, \quad\left\|\boldsymbol{u}^{\varepsilon}-\boldsymbol{u}^{0}\right\|_{L^{2}\left(0, T ; H^{1}(\Omega)\right)} \leq \kappa_{T} \varepsilon^{\frac{1}{2}},
$$

for a positive constant $\kappa_{T}:=\kappa_{T}\left(T, \boldsymbol{u}_{0}, \boldsymbol{f}, \Omega\right)$ depending on the data, but independent of $\varepsilon$ (see also Remark 4.1).

REMARK 2.3. The regularity assumptions (1.6) or (1.7) are not the minimal requirement for the analysis in this article. For instance, $(1.6)_{1}$ can be replaced by

$$
\boldsymbol{u}_{0} \in H \cap H^{3}(\Gamma)
$$

In this article, we use the notation conventions below.

\section{Notation 2.4 .}

$\kappa:=\kappa\left(\boldsymbol{u}_{0}, \boldsymbol{f}, \Omega, \sigma\right)$ is a constant depending on the data, but independent of $\varepsilon$ or $t$,

where $\sigma$, defined in (4.11), is a smooth (of class $C^{\infty}$ ) cut-off function.

Notation 2.5. e.s.t is a function (or a constant) whose norm in all Sobolev spaces $H^{s}$ (and thus spaces $C^{s}$ ) is exponentially small with a bound of the form $c_{1} e^{-c_{2} / \varepsilon^{\gamma}}$, $c_{1}, c_{2}, \gamma>0$, for each $s$.

Notation 2.6. Near the boundary $\Gamma$, we write

$$
\frac{\partial}{\partial \tau}:=\left(\text { any tangential derivative in } \xi_{1} \text { or } \xi_{2}\right)
$$

where $\xi_{1}$ and $\xi_{2}$ are the tangential variables near $\Gamma$ as defined in Section 3.

Now, using the asymptotic expansion of the Stokes solution, defined in (4.1), we state the main result.

THEOREM 2.7. Under the assumptions (1.6) and (1.7), the difference between the Stokes solution $\boldsymbol{u}^{\varepsilon}$ and its asymptotic expansion $\boldsymbol{u}^{0}+\boldsymbol{\theta}^{0}$ satisfies the estimates

$$
\left\|\boldsymbol{u}^{\varepsilon}-\left(\boldsymbol{u}^{0}+\boldsymbol{\theta}^{0}\right)\right\|_{L^{\infty}\left(0, T ; L^{2}(\Omega)\right)} \leq \kappa_{T} \varepsilon^{\frac{1}{2}}, \quad\left\|\boldsymbol{u}^{\varepsilon}-\left(\boldsymbol{u}^{0}+\boldsymbol{\theta}^{0}\right)\right\|_{L^{2}\left(0, T ; H^{1}(\Omega)\right)} \leq \kappa_{T},
$$

for a positive constant $\kappa_{T}:=\kappa_{T}\left(T, \boldsymbol{u}_{0}, \boldsymbol{f}, \Omega, \sigma\right)$ depending on the data, but independent of $\varepsilon$. Moreover, as the viscosity $\varepsilon$ tends to zero, we have the uniform $L^{2}$ convergence of the Stokes solution $\boldsymbol{u}^{\varepsilon}$ to the linearized Euler solution $\boldsymbol{u}^{0}$, in the sense that

$$
\left\|\boldsymbol{u}^{\varepsilon}-\boldsymbol{u}^{0}\right\|_{L^{\infty}\left(0, T ; L^{2}(\Omega)\right)} \leq \kappa_{T} \varepsilon^{\frac{1}{4}} .
$$




\section{Curvilinear system}

We let $\boldsymbol{x}=\left(x_{1}, x_{2}, x_{3}\right)$ denote the Cartesian coordinates of a point in $\mathbb{R}^{3}$.

To avoid some technical difficulties of geometry, we assume that the smooth boundary $\Gamma$ satisfying (1.7) is a $2 \mathrm{D}$ compact manifold in $\mathbb{R}^{3}$ having no umbilical points; namely, at each point on $\Gamma$, the two principal curvatures are different. Then, thanks to Lemma 3.6.6 of [14], we can construct a curvilinear system globally on the boundary $\Gamma$ in which the metric tensor is diagonal and the coordinate lines at each point are parallel to the principal directions. Such a coordinate system is called the principal curvature coordinate system. As explained in, e.g., [8], this technical assumption on $\Gamma$ is only for the simplicity of the display below, and it can be omitted by considering an atlas of finitely many charts on $\Gamma$.

Inside of a tubular neighborhood $\Omega_{3 \delta}$ with a small, but fixed, width $3 \delta>0$, we extend the principal curvature coordinates on $\Gamma$ in the direction of $-\boldsymbol{n}$, where $\boldsymbol{n}$ is the outer unit normal vector on $\Gamma$. As a result, we obtain a triply orthogonal coordinate system $\boldsymbol{\xi}$ in $\mathbb{R}_{\boldsymbol{\xi}}^{3}$, such that $\Omega_{3 \delta}$ is diffeomorphic to

$$
\Omega_{3 \delta, \boldsymbol{\xi}}:=\left\{\boldsymbol{\xi}=\left(\xi^{\prime}, \xi_{3}\right) \in \mathbb{R}_{\boldsymbol{\xi}}^{3} \mid \xi^{\prime}=\left(\xi_{1}, \xi_{2}\right) \in \omega_{\xi^{\prime}}, 0<\xi_{3}<3 \delta\right\},
$$

for some bounded set $\omega_{\xi^{\prime}}$ in $\mathbb{R}_{\xi^{\prime}}^{2}$. Then $\xi_{3}$ measures the distance from a point in $\Omega_{3 \delta}$ to $\Gamma$, and

$$
\Gamma=\left\{\boldsymbol{\xi} \in \mathbb{R}_{\boldsymbol{\xi}}^{3} \mid \xi^{\prime}=\left(\xi_{1}, \xi_{2}\right) \in \omega_{\xi^{\prime}}, \xi_{3}=0\right\} .
$$

In (3.1) and (3.2), with a slight abuse of notation, we use the subscript $\boldsymbol{\xi}$ to denote the variable in space.

Using the covariant basis $\boldsymbol{g}_{i}=\partial \boldsymbol{x} / \partial \xi_{i}, 1 \leq i \leq 3$, we write the metric tensor of $\boldsymbol{\xi}$,

$$
\begin{aligned}
\left(g_{i j}\right)_{1 \leq i, j \leq 3} & :=\left(\boldsymbol{g}_{i} \cdot \boldsymbol{g}_{j}\right)_{1 \leq i, j \leq 3} \\
& =\left(\begin{array}{ccc}
{\left[1-\kappa_{1}\left(\xi^{\prime}\right) \xi_{3}\right]^{2} \widetilde{g}_{11}\left(\xi^{\prime}\right)} & 0 & 0 \\
0 & {\left[1-\kappa_{2}\left(\xi^{\prime}\right) \xi_{3}\right]^{2} \widetilde{g}_{22}\left(\xi^{\prime}\right)} & 0 \\
0 & 0 & 1
\end{array}\right),
\end{aligned}
$$

where $\kappa_{i}\left(\xi^{\prime}\right), i=1,2$, is the principal curvature in the principal direction $\boldsymbol{g}_{i}$ on $\Gamma$.

By the choice of the small thickness $3 \delta>0$, we have

$$
g(\boldsymbol{\xi}):=\operatorname{det}\left(g_{i j}\right)_{1 \leq i, j \leq 3}>0 \text { for all } \boldsymbol{\xi} \text { in the closure of } \Omega_{3 \delta, \boldsymbol{\xi}}
$$

We introduce the normalized covariant vectors,

$$
\boldsymbol{e}_{i}=\frac{\boldsymbol{g}_{\boldsymbol{i}}}{\left|\boldsymbol{g}_{i}\right|}, 1 \leq i \leq 3
$$

and set

$$
h_{i}(\boldsymbol{\xi})=\sqrt{g_{i i}}, i=1,2, \quad h(\boldsymbol{\xi})=\sqrt{g} .
$$

The function $h(\boldsymbol{\xi})>0$ is the magnitude of the Jacobian determinant for the transformation from $\boldsymbol{x}$ to $\boldsymbol{\xi}$.

For a vector valued function $F$, defined in $\Omega_{3 \delta, \xi}$, in the form

$$
F=\sum_{i=1}^{3} F^{i}(\boldsymbol{\xi}) \boldsymbol{e}_{i}
$$


one can classically express the divergence operator acting on $F$ in the $\boldsymbol{\xi}$ variables (see [2] or [14]) as

$$
\operatorname{div} F=\frac{1}{h} \sum_{i=1}^{2} \frac{\partial}{\partial \xi_{i}}\left(\frac{h}{h_{i}} F^{i}\right)+\frac{1}{h} \frac{\partial\left(h F^{3}\right)}{\partial \xi_{3}} .
$$

The Laplacian of $F$ is given in the form

$$
\Delta F=\sum_{i=1}^{3}\left(\mathcal{S}^{i} F+\mathcal{L} F^{i}+\frac{\partial^{2} F^{i}}{\partial \xi_{3}^{2}}\right) \boldsymbol{e}_{i},
$$

where

$$
\left\{\begin{array}{l}
\mathcal{S}^{i} F=\left(\begin{array}{l}
\text { linear combination of tangential derivatives } \\
\text { of } F^{j}, 1 \leq j \leq 3, \text { in } \xi^{\prime}, \text { up to order } 2
\end{array}\right), \\
\mathcal{L} F^{i}=\left(\text { proportional to } \frac{\partial F^{i}}{\partial \xi_{3}}\right)
\end{array}\right.
$$

REMARK 3.1. Note that the coefficients of $\mathcal{S}^{i}, 1 \leq i \leq 3$, and $\mathcal{L}$ are multiples of $h$, $1 / h, h_{i}, 1 / h_{i}, i=1,2$, and their derivatives. Thanks to (3.4), all these quantities are well-defined because of the regularity assumption (1.7) on $\partial \Omega$. The explicit expression of (3.8) appears in, e.g., [5]. (The expression of $\mathcal{L}^{i}, 1 \leq i \leq 3$, in [5] does not depend on $i$, and hence, in (3.9), we drop the superscript $i$.)

\section{Asymptotic expansion at order $\varepsilon^{0}$}

To study the asymptotic behavior of $\boldsymbol{u}^{\varepsilon}$, the solution of (1.2), as the viscosity $\varepsilon$ tends to zero, we propose an asymptotic expansion, at order $\varepsilon^{0}$ of $\left(\boldsymbol{u}^{\varepsilon}, p^{\varepsilon}\right)$ in the form

$$
\boldsymbol{u}^{\varepsilon} \simeq \boldsymbol{u}^{0}+\boldsymbol{\Theta}^{0}, \quad p^{\varepsilon} \simeq p^{0} .
$$

Here $\left(\boldsymbol{u}^{0}, p^{0}\right)$ is the solution of (1.5), and $\boldsymbol{\Theta}^{0}$ is a divergence-free corrector which balances the difference $\boldsymbol{u}^{\varepsilon}-\boldsymbol{u}^{0}$ on the boundary $\Gamma$; it will be constructed below in the form

$$
\boldsymbol{\Theta}^{0}=\sum_{i=1}^{3} \Theta^{0, i} \boldsymbol{e}_{i}
$$

We formally insert $\boldsymbol{\Theta}^{0} \simeq \boldsymbol{u}^{\varepsilon}-\boldsymbol{u}^{0}$ into the difference of the equations (1.2) and (1.5), and find

$$
\begin{cases}\frac{\partial \boldsymbol{\Theta}^{0}}{\partial t}-\varepsilon \Delta \boldsymbol{\Theta}^{0}+\nabla\left(p^{\varepsilon}-p^{0}\right)=\varepsilon \Delta \boldsymbol{u}^{0}, & \Omega \times(0, T), \\ \operatorname{div} \boldsymbol{\Theta}^{0}=0, & \Omega \times(0, T), \\ \boldsymbol{\Theta}^{0}=-\boldsymbol{u}^{0}, & \Gamma \times(0, T), \\ \left.\boldsymbol{\Theta}^{0}\right|_{t=0}=0, & \Omega .\end{cases}
$$

To extract the essential parts of (4.3), we follow the approach of Prandtl in, e.g., [23].

Recalling that $\xi_{3}$ (or $\xi_{i}, i=1,2$,) is the normal (or tangential) variable near the boundary $\Gamma$, we first use the Prandtl theory to find that the thickness of the boundary 
layers with respect to the viscosity is $\varepsilon^{1 / 2}$. Then, using the ansatz $\partial / \partial \xi_{i} \simeq \sqrt{\varepsilon}\left(\partial / \partial \xi_{3}\right)$, $i=1,2$, and using (3.7) and (4.3) , we notice that $\Theta^{0, i} \simeq \sqrt{\varepsilon} \Theta^{0,3}, i=1,2$. Using these observations, we write the equation (4.3) in the $\boldsymbol{\xi}$ variables, and collect the leading order terms of order $\varepsilon^{0}$. For $i=1,2$,

$$
\begin{cases}\frac{\partial \Theta^{0, i}}{\partial t}-\varepsilon \frac{\partial^{2} \Theta^{0, i}}{\partial \xi_{3}^{2}}=0, & \text { in (at least) } \Omega_{3 \delta} \times(0, T) \\ \Theta^{0, i}=-\widetilde{u}^{0, i}, & \Gamma \times(0, T), \text { i.e., at } \xi_{3}=0 \\ \left.\Theta^{0, i}\right|_{t=0}=0, & \Omega,\end{cases}
$$

and

$$
\left\{\begin{array}{lr}
\sum_{i=1}^{2} \frac{\partial}{\partial \xi_{1}}\left(\frac{h}{h_{i}} \Theta^{0, i}\right)+\frac{\partial\left(h \Theta^{0,3}\right)}{\partial \xi_{3}}=0, & \text { in (at least) } \Omega_{3 \delta} \times(0, T), \\
\Theta^{0,3}=0, & \Gamma \times(0, T), \text { i.e., at } \xi_{3}=0, \\
\left.\Theta^{0,3}\right|_{t=0}=0, & \Omega,
\end{array}\right.
$$

where

$$
\widetilde{u}^{0, i}=\widetilde{u}^{0, i}\left(\xi^{\prime} ; t\right):=\left.\left(\boldsymbol{u}^{0} \cdot \boldsymbol{e}_{i}\right)\right|_{\xi_{3}=0} .
$$

We define the complimentary error function on $\mathbb{R}$,

$$
\operatorname{erfc}(z):=\frac{1}{\sqrt{2 \pi}} \int_{z}^{\infty} e^{-y^{2} / 2} d y
$$

which satisfies

$$
\operatorname{erfc}(0)=\frac{1}{2}, \quad \operatorname{erfc}(\infty)=0
$$

From [1], we recall that, for $i=1,2$,

$$
\bar{\theta}^{0, i}(\boldsymbol{\xi} ; t)=-2 \widetilde{u}^{0, i}\left(\xi^{\prime} ; 0\right) \operatorname{erfc}\left(\frac{\xi_{3}}{\sqrt{2 \varepsilon t}}\right)-2 \int_{0}^{t} \frac{\partial \widetilde{u}^{0, i}}{\partial t}\left(\xi^{\prime} ; s\right) \operatorname{erfc}\left(\frac{\xi_{3}}{\sqrt{2 \varepsilon(t-s)}}\right) d s,
$$

is a solution of the heat equation on the half plane,

$$
\begin{cases}\frac{\partial \bar{\theta}^{0, i}}{\partial t}-\varepsilon \frac{\partial^{2} \bar{\theta}^{0, i}}{\partial \xi_{3}^{2}}=0, & \xi_{3}, t>0, \\ \bar{\theta}^{0, i}=-\widetilde{u}^{0, i}, & \xi_{3}=0, t>0, \\ \bar{\theta}^{0, i} \rightarrow 0, & \text { as } \xi_{3} \rightarrow \infty, \\ \left.\bar{\theta}^{0, i}\right|_{t=0}=0, & \xi_{3}>0 .\end{cases}
$$

We introduce a truncation function $\sigma=\sigma\left(\xi_{3}\right)$, of class $C^{\infty}$, such that

$$
\sigma\left(\xi_{3}\right)=\left\{\begin{array}{l}
1,0 \leq \xi_{3} \leq \delta \\
0, \xi_{3} \geq 2 \delta
\end{array}\right.
$$


Using (4.9) and (4.11), we define the tangential component $\Theta^{0, i}, i=1,2$, of $\mathbf{\Theta}^{0}$ in (4.2) in the form

$$
\begin{aligned}
& \Theta^{0, i}(\boldsymbol{\xi} ; t) \\
= & -\left.2 \widetilde{u}^{0, i}\left(\xi^{\prime} ; 0\right) \frac{h_{i}}{h}(\boldsymbol{\xi}) \frac{h}{h_{i}}\right|_{\xi_{3}=0} \frac{\partial}{\partial \xi_{3}}\left\{\sigma\left(\xi_{3}\right) \int_{0}^{\xi_{3}} \operatorname{erfc}\left(\frac{\eta}{\sqrt{2 \varepsilon t}}\right) d \eta\right\} \\
& -\left.2 \int_{0}^{t} \frac{\partial \widetilde{u}^{0, i}}{\partial t}\left(\xi^{\prime} ; s\right) \frac{h_{i}}{h}(\boldsymbol{\xi}) \frac{h}{h_{i}}\right|_{\xi_{3}=0} \frac{\partial}{\partial \xi_{3}}\left\{\sigma\left(\xi_{3}\right) \int_{0}^{\xi_{3}} \operatorname{erfc}\left(\frac{\eta}{\sqrt{2 \varepsilon(t-s)}}\right) d \eta\right\} d s .
\end{aligned}
$$

Then, using $(4.5)_{1}$ and (4.12), we define the normal component $\Theta^{0,3}$ :

$$
\begin{aligned}
& \Theta^{0,3}(\boldsymbol{\xi} ; t) \\
= & \frac{1}{h(\boldsymbol{\xi})} \sum_{i=1}^{2} \frac{\partial}{\partial \xi_{i}}\left\{\left.\widetilde{u}^{0, i}\left(\xi^{\prime} ; 0\right) \frac{h}{h_{i}}\right|_{\xi_{3}=0}\right\} \sigma\left(\xi_{3}\right) \int_{0}^{\xi_{3}} \operatorname{erfc}\left(\frac{\eta}{\sqrt{2 \varepsilon t}}\right) d \eta \\
& +2 \frac{1}{h(\boldsymbol{\xi})} \int_{0}^{t}\left[\sum_{i=1}^{2} \frac{\partial}{\partial \xi_{i}}\left\{\left.\frac{\partial \widetilde{u}^{0, i}}{\partial t}\left(\xi^{\prime} ; s\right) \frac{h}{h_{i}}\right|_{\xi_{3}=0}\right\} \sigma\left(\xi_{3}\right) \int_{0}^{\xi_{3}} \operatorname{erfc}\left(\frac{\eta}{\sqrt{2 \varepsilon(t-s)}}\right) d \eta\right] d s .
\end{aligned}
$$

REMark 4.1. If the initial data $\boldsymbol{u}_{0}$ is in $V\left(\left.\boldsymbol{u}_{0}\right|_{\Gamma}=0\right)$, then the first part of $\Theta^{0, i}$, $1 \leq i \leq 3$, which is not involved in the time integration, is identically zero. Thus we notice that the first part of the corrector is related to the boundary layer produced by the non-compatible initial data. On the other hand, as mentioned in Remark 2.2, when $\boldsymbol{f} \equiv 0$, the linearized Euler solution $\boldsymbol{u}^{0}$ is stationary as $\boldsymbol{u}_{0}$. Then, in this case, the second part of the corrector, which is involved in the time integration, vanishes. As an extreme case, if $\boldsymbol{u}_{0} \in V$ and $\boldsymbol{f} \equiv 0$, then $\boldsymbol{\Theta}^{0} \equiv 0$. Therefore, without using any corrector function, one can verify (2.6); no boundary layers occur in this case.

Applying (4.12) and (4.13) to (3.7), we notice that

$$
\operatorname{div} \Theta^{0}=0 .
$$

Using (4.8) and (4.11), we see that $\Theta^{0}$, defined by (4.12) and (4.13), satisfies the desired boundary and initial conditions in (4.4) and (4.5). Moreover, $\Theta^{0}$ and its derivatives vanish outside of $\Omega_{2 \delta}$ :

$$
\left.\frac{\partial^{k+m+1} \boldsymbol{\Theta}^{0}}{\partial t \partial \tau^{k} \partial \xi_{3}^{m}}\right|_{\xi_{3} \geq 2 \delta}=0, \quad 0 \leq k, m \leq 2
$$

From (4.7), one can verify that, for $z>0$,

$$
|\operatorname{erfc}(z)| \leq \frac{1}{2} e^{-z^{2} / 2} \quad \text { (pointwise) }
$$


and hence we find that, for $\xi_{3}, t>0$,

$$
\left\{\begin{aligned}
\left|\int_{0}^{\infty} \operatorname{erfc}\left(\frac{\eta}{\sqrt{2 \varepsilon t}}\right) d \eta\right| & \leq \frac{1}{2} \int_{0}^{\infty} e^{-\eta^{2} /(4 \varepsilon t)} d \eta \leq \kappa \varepsilon^{\frac{1}{2}} t^{\frac{1}{2}} \\
\left|\int_{\xi_{3}}^{\infty} \operatorname{erfc}\left(\frac{\eta}{\sqrt{2 \varepsilon t}}\right) d \eta\right| & \leq \frac{1}{2} \int_{\xi_{3}}^{\infty} e^{-\eta^{2} /(4 \varepsilon t)} d \eta \\
& \leq \kappa e^{-\xi_{3}^{2} /(8 \varepsilon t)} \int_{0}^{\infty} e^{-\eta^{2} /(8 \varepsilon t)} d \eta \\
& \leq \kappa \varepsilon^{\frac{1}{2}} t^{\frac{1}{2}} e^{-\xi_{3}^{2} /(8 \varepsilon t)}
\end{aligned}\right.
$$

Using (4.9), (4.12), (4.13), and (4.16), we write $\Theta^{0}$ in the form

$$
\boldsymbol{\Theta}^{0}=\boldsymbol{\theta}^{0}+\boldsymbol{\varphi}^{0}+\text { e.s.t. }, \quad \boldsymbol{\theta}^{0}=\sum_{i=1}^{3} \theta^{0, i} \boldsymbol{e}_{i}, \quad \boldsymbol{\varphi}^{0}=\sum_{i=1}^{3} \varphi^{0, i} \boldsymbol{e}_{i},
$$

where

$$
\left\{\begin{aligned}
\theta^{0, i}= & \left.\frac{h_{i}}{h}(\boldsymbol{\xi}) \frac{h}{h_{i}}\right|_{\xi_{3}=0} \sigma\left(\xi_{3}\right) \bar{\theta}^{0, i}, \quad i=1,2, \\
\theta^{0,3}= & -2 \frac{1}{h(\boldsymbol{\xi})} \sigma\left(\xi_{3}\right) \sum_{i=1}^{2} \frac{\partial}{\partial \xi_{i}}\left\{\left.\widetilde{u}^{0, i}\left(\xi^{\prime} ; 0\right) \frac{h}{h_{i}}\right|_{\xi_{3}=0}\right\} \int_{\xi_{3}}^{\infty} \operatorname{erfc}\left(\frac{\eta}{\sqrt{2 \varepsilon t}}\right) d \eta \\
& -2 \frac{1}{h(\boldsymbol{\xi})} \sigma\left(\xi_{3}\right) \int_{0}^{t}\left[\sum_{i=1}^{2} \frac{\partial}{\partial \xi_{i}}\left\{\left.\frac{\partial \widetilde{u}^{0, i}}{\partial t}\left(\xi^{\prime} ; s\right) \frac{h}{h_{i}}\right|_{\xi_{3}=0}\right\} \int_{\xi_{3}}^{\infty} \operatorname{erfc}\left(\frac{\eta}{\sqrt{2 \varepsilon(t-s)}}\right) d \eta\right] d s
\end{aligned}\right.
$$

and

$$
\left\{\begin{aligned}
\varphi^{0, i} & =-\left.2 \widetilde{u}^{0, i}\left(\xi^{\prime} ; 0\right) \frac{h_{i}}{h}(\boldsymbol{\xi}) \frac{h}{h_{i}}\right|_{\xi_{3}=0} \sigma^{\prime}\left(\xi_{3}\right) \int_{0}^{\infty} \operatorname{erfc}\left(\frac{\eta}{\sqrt{2 \varepsilon t}}\right) d \eta \\
& -2 \sigma^{\prime}\left(\xi_{3}\right) \int_{0}^{t}\left[\left.\frac{\partial \widetilde{u}^{0, i}}{\partial t}\left(\xi^{\prime} ; s\right) \frac{h_{i}}{h}(\boldsymbol{\xi}) \frac{h}{h_{i}}\right|_{\xi_{3}=0} \int_{0}^{\infty} \operatorname{erfc}\left(\frac{\eta}{\sqrt{2 \varepsilon(t-s)}}\right) d \eta\right] d s \\
\varphi^{0,3} & =2 \frac{1}{h(\boldsymbol{\xi})} \sigma\left(\xi_{3}\right) \sum_{i=1}^{2} \frac{\partial}{\partial \xi_{i}}\left\{\left.\widetilde{u}^{0, i}\left(\xi^{\prime} ; 0\right) \frac{h}{h_{i}}\right|_{\xi_{3}=0}\right\} \int_{0}^{\infty} \operatorname{erfc}\left(\frac{\eta}{\sqrt{2 \varepsilon t}}\right) d \eta \\
& +2 \frac{1}{h(\boldsymbol{\xi})} \sigma\left(\xi_{3}\right) \int_{0}^{t}\left[\sum_{i=1}^{2} \frac{\partial}{\partial \xi_{i}}\left\{\left.\frac{\partial \widetilde{u}^{0, i}}{\partial t}\left(\xi^{\prime} ; s\right) \frac{h}{h_{i}}\right|_{\xi_{3}=0}\right\} \int_{0}^{\infty} \operatorname{erfc}\left(\frac{\eta}{\sqrt{2 \varepsilon(t-s)}}\right) d \eta\right] d s .
\end{aligned}\right.
$$

From (4.18) and (4.19), it is easy to see that $\boldsymbol{\theta}^{0}, \boldsymbol{\varphi}^{0}$, and their derivatives vanish outside of $\Omega_{2 \delta}$ :

$$
\left.\frac{\partial^{k+m+1} \boldsymbol{\theta}^{0}}{\partial t \partial \tau^{k} \partial \xi_{3}^{m}}\right|_{\xi_{3} \geq 2 \delta}=0,\left.\quad \frac{\partial^{k+m+1} \varphi^{0}}{\partial t \partial \tau^{k} \partial \xi_{3}^{m}}\right|_{\xi_{3} \geq 2 \delta}=0, \quad 0 \leq k, m \leq 2 .
$$

4.1. Estimates on the corrector. In this section, we aim to estimate the corrector $\Theta^{0}$ defined in (4.2). Toward this end, thanks to (4.17), we first notice that it is enough to estimate $\boldsymbol{\theta}^{0}$ and $\varphi^{0}$, and we sequentially estimate $\boldsymbol{\theta}^{0}$ and $\varphi^{0}$ in the lemmas below. 
We state and prove a simple lemma below.

Lemma 4.2. For any $\alpha, p \geq 0$ and $q \geq 1$, we have

$$
\left\|t^{\alpha}\left(\frac{\xi_{3}}{\sqrt{\varepsilon t}}\right)^{p} e^{-\xi_{3}^{2} /(4 q \varepsilon t)}\right\|_{L^{2}\left(\Omega_{3 \delta}\right)} \leq \kappa t^{\alpha+\frac{1}{4}} \varepsilon^{\frac{1}{4}}
$$

Proof.

$$
\begin{aligned}
\| t^{\alpha}\left(\frac{\xi_{3}}{\sqrt{\varepsilon t}}\right)^{p} e^{-\xi_{3}^{2} /(4 q \varepsilon t) \|_{L^{2}\left(\Omega_{3 \delta}\right)}^{2}} & \leq \int_{\omega^{\prime}} \int_{0}^{3 \delta} t^{2 \alpha}\left(\frac{\xi_{3}}{\sqrt{\varepsilon t}}\right)^{2 p} e^{-\xi_{3}^{2} /(2 q \varepsilon t)} h(\boldsymbol{\xi}) d \xi_{3} d \xi^{\prime} \\
& \leq\left(\operatorname{setting} \eta=\xi_{3} / \sqrt{q \varepsilon t}, \text { and using }|h| \leq \kappa\right) \\
& \leq \kappa \int_{\omega^{\prime}} \int_{0}^{\infty} t^{2 \alpha} \sqrt{\varepsilon t} \eta^{2 p} e^{-\eta^{2} / 2} d \eta d \xi^{\prime} \\
& \leq \kappa t^{2 \alpha+\frac{1}{2}} \varepsilon^{\frac{1}{2}}
\end{aligned}
$$

Thanks to Lemma 4.2, we prove the $L^{2}$ estimates on $\bar{\theta}^{0, i}, i=1,2$, and its derivatives.

LEMMA 4.3. Under the regularity assumptions (1.6) and (1.7), $\bar{\theta}^{0, i}$, which is defined in (4.9), satisfies

$$
\left\|\frac{\partial^{k+m} \bar{\theta}^{0, i}}{\partial \tau^{k} \partial \xi_{3}^{m}}\right\|_{L^{2}\left(\Omega_{3 \delta}\right)} \leq \kappa\left(t^{\frac{1}{4}}+t^{\frac{5}{4}}\right) t^{-\frac{m}{2}} \varepsilon^{\frac{1}{4}-\frac{m}{2}}, \quad i=1,2,0 \leq k \leq 2, m=0,1 .
$$

Proof. We differentiate (4.9) and write

$$
\begin{aligned}
\frac{\partial^{k+m} \bar{\theta}^{0, i}}{\partial \tau^{k} \xi_{3}^{m}}= & -2 \frac{\partial^{k} \widetilde{u}^{0, i}}{\partial \tau^{k}}\left(\xi^{\prime} ; 0\right) \frac{\partial^{m}}{\partial \xi_{3}^{m}}\left\{\operatorname{erfc}\left(\frac{\xi_{3}}{\sqrt{2 \varepsilon t}}\right)\right\} \\
& -2 \int_{0}^{t} \frac{\partial^{k} \widetilde{u}^{0, i}}{\partial \tau^{k}}\left(\xi^{\prime} ; s\right) \frac{\partial^{m}}{\partial \xi_{3}^{m}}\left\{\operatorname{erfc}\left(\frac{\xi_{3}}{\sqrt{2 \varepsilon(t-s)}}\right)\right\} d s, \quad k, m \geq 0 .
\end{aligned}
$$

Moreover, using (4.7) and (4.15), we find that, for $\xi_{3}, t>0$,

$$
\left\{\begin{array}{l}
\left|\operatorname{erfc}\left(\frac{\xi_{3}}{\sqrt{2 \varepsilon t}}\right)\right| \leq \kappa e^{-\xi_{3}^{2} /(4 \varepsilon t)} \\
\left|\frac{\partial}{\partial \xi_{3}}\left\{\operatorname{erfc}\left(\frac{\xi_{3}}{\sqrt{2 \varepsilon t}}\right)\right\}\right| \leq \kappa\left(t^{-\frac{1}{2}}+t^{\frac{1}{2}}\right) \varepsilon^{-\frac{1}{2}} e^{-\xi_{3}^{2} /(4 \varepsilon t)} .
\end{array}\right.
$$

Then, from Lemma 4.2, (4.23), and (4.24), (4.22) follows.

Now, from $(4.18)_{1}$ and Lemma 4.3 , we deduce the $L^{2}$ estimates on the tangential component $\theta^{0, i}, i=1,2$, of $\boldsymbol{\theta}^{0}$, and its derivatives:

$$
\left\|\frac{\partial^{k+m} \theta^{0, i}}{\partial \tau^{k} \partial \xi_{3}^{m}}\right\|_{L^{2}\left(\Omega_{3 \delta}\right)} \leq \kappa\left(t^{\frac{1}{4}-\frac{m}{2}}+t^{\frac{5}{4}}\right) \varepsilon^{\frac{1}{4}-\frac{m}{2}}, \quad i=1,2,0 \leq k \leq 2, m=0,1 .
$$

We state and prove the estimates on the normal component $\theta^{0,3}$ of $\boldsymbol{\theta}^{0}$. 
Lemma 4.4. Under the regularity assumptions (1.6) and (1.7), $\theta^{0,3}$, which is defined in $(4.18)_{2}$, satisfies

$$
\left\{\begin{array}{l}
\left\|\frac{\partial \theta^{0,3}}{\partial t}\right\|_{L^{2}\left(\Omega_{3 \delta}\right)} \leq \kappa\left(t^{-\frac{1}{4}}+t^{\frac{3}{4}}\right) \varepsilon^{\frac{3}{4}} \\
\left\|\frac{\partial^{k+m} \theta^{0,3}}{\partial \tau^{k} \partial \xi_{3}^{m}}\right\|_{L^{2}\left(\Omega_{3 \delta}\right)} \leq \kappa\left(t^{\frac{3}{4}-\frac{m}{2}}+t^{\frac{7}{4}}\right) \varepsilon^{\frac{3}{4}-\frac{m}{2}}, \quad 0 \leq k, m \leq 2 .
\end{array}\right.
$$

Proof. To verify $(4.26)_{1}$, using (4.8), we differentiate $(4.18)_{2}$ in $t$ and write

$$
\left|\frac{\partial \theta^{0,3}}{\partial t}\right| \leq \kappa \int_{\xi_{3}}^{\infty}\left|\frac{\partial}{\partial t}\left\{\operatorname{erfc}\left(\frac{\eta}{\sqrt{2 \varepsilon t}}\right)\right\}\right| d \eta+\kappa \int_{0}^{t} \int_{\xi_{3}}^{\infty}\left|\frac{\partial}{\partial t}\left\{\operatorname{erfc}\left(\frac{\eta}{\sqrt{2 \varepsilon(t-s)}}\right)\right\}\right| d \eta d s .
$$

On the other hand, from (4.7), we find that

$$
\left|\frac{\partial}{\partial t}\left\{\operatorname{erfc}\left(\frac{\eta}{\sqrt{2 \varepsilon t}}\right)\right\}\right| \leq \kappa t^{-\frac{3}{2}} \varepsilon^{-\frac{1}{2}} \eta e^{-\eta^{2} /(4 \varepsilon t)},
$$

and hence,

$$
\begin{aligned}
\int_{\xi_{3}}^{\infty}\left|\frac{\partial}{\partial t}\left\{\operatorname{erfc}\left(\frac{\eta}{\sqrt{2 \varepsilon t}}\right)\right\}\right| d \eta & \leq \kappa t^{-1} \int_{\xi_{3}}^{\infty} \frac{\eta}{\sqrt{\varepsilon t}} e^{-\eta^{2} /(4 \varepsilon t)} d \eta \\
& \leq \kappa t^{-1} e^{-\xi_{3}^{2} /(8 \varepsilon t)} \int_{0}^{\infty} \frac{\eta}{\sqrt{\varepsilon t}} e^{-\eta^{2} /(8 \varepsilon t)} d \eta \\
& \leq \kappa t^{-\frac{1}{2}} \varepsilon^{\frac{1}{2}} e^{-\xi_{3}^{2} /(8 \varepsilon t)}
\end{aligned}
$$

Then, from (4.27), (4.29), and Lemma 4.2, (4.26) 1 follows.

To show $(4.26)_{2}$, using $(4.18)_{2}$, we write, for $0 \leq k \leq 2$ and $m \geq 0$,

$$
\begin{aligned}
& \quad\left|\frac{\partial^{k+m} \theta^{0,3}}{\partial \tau^{k} \partial \xi_{3}^{m}}\right| \\
& \leq \kappa \int_{\xi_{3}}^{\infty}\left|\operatorname{erfc}\left(\frac{\eta}{\sqrt{2 \varepsilon t}}\right)\right| d \eta+\kappa \sum_{l=0}^{m-1}\left|\frac{\partial^{l}}{\partial \xi_{3}^{l}}\left\{\operatorname{erfc}\left(\frac{\xi_{3}}{\sqrt{2 \varepsilon t}}\right)\right\}\right| \\
& \quad+\kappa \int_{0}^{t}\left[\int_{\xi_{3}}^{\infty}\left|\operatorname{erfc}\left(\frac{\eta}{\sqrt{2 \varepsilon(t-s)}}\right)\right| d \eta+\sum_{l=0}^{m-1}\left|\frac{\partial^{l}}{\partial \xi_{3}^{l}}\left\{\operatorname{erfc}\left(\frac{\xi_{3}}{\sqrt{2 \varepsilon(t-s)}}\right)\right\}\right|\right] d s .
\end{aligned}
$$

Then, using (4.16) and (4.24), we find that, for $0 \leq k \leq 2$,

$$
\left\{\begin{array}{l}
\left|\frac{\partial^{k} \theta^{0,3}}{\partial \tau^{k}}\right| \leq \kappa\left(t^{\frac{1}{2}}+t^{\frac{3}{2}}\right) \varepsilon^{\frac{1}{2}} e^{-\xi_{3}^{2} /(4 \varepsilon t)} \\
\left|\frac{\partial^{k+1} \theta^{0,3}}{\partial \tau^{k} \partial \xi_{3}}\right| \leq \kappa\left\{\left(t^{\frac{1}{2}}+t^{\frac{3}{2}}\right) \varepsilon^{\frac{1}{2}}+(1+t)\right\} e^{-\xi_{3}^{2} /(4 \varepsilon t)} \\
\left|\frac{\partial^{k+2} \theta^{0,3}}{\partial \tau^{k} \partial \xi_{3}^{2}}\right| \leq \kappa\left\{\left(t^{\frac{1}{2}}+t^{\frac{3}{2}}\right) \varepsilon^{\frac{1}{2}}+(1+t)+\left(t^{-\frac{1}{2}}+t^{\frac{1}{2}}\right) \varepsilon^{-\frac{1}{2}}\right\} e^{-\xi_{3}^{2} /(4 \varepsilon t)}
\end{array}\right.
$$

From (4.31) and Lemma 4.2, $(4.26)_{2}$ follows, and hence the proof of Lemma 4.4 is complete. 
Finally, we state and prove the estimates on the supplementary part $\varphi^{0}$ of the corrector $\boldsymbol{\Theta}^{0}$.

Lemma 4.5. Under the regularity assumptions (1.6) and (1.7), $\varphi^{0}$, which is defined in (4.19), satisfies

$$
\left\{\begin{array}{l}
\left\|\frac{\partial \varphi^{0}}{\partial t}\right\|_{L^{2}\left(\Omega_{3 \delta}\right)} \leq \kappa\left(t^{-\frac{1}{2}}+t^{\frac{1}{2}}\right) \varepsilon^{\frac{1}{2}} \\
\left\|\frac{\partial^{k+m} \varphi^{0}}{\partial \tau^{k} \partial \xi_{3}^{m}}\right\|_{L^{2}\left(\Omega_{3 \delta}\right)} \leq \kappa\left(t^{\frac{1}{2}}+t^{\frac{3}{2}}\right) \varepsilon^{\frac{1}{2}}, \quad 0 \leq k, m \leq 2 .
\end{array}\right.
$$

Proof. For (4.32) 1 , using (4.8), (4.19), and (4.28), we find that, for $1 \leq i \leq 3$,

$$
\begin{aligned}
\left|\frac{\partial \varphi^{0, i}}{\partial t}\right| & \leq \kappa \int_{0}^{\infty}\left|\frac{\partial}{\partial t}\left\{\operatorname{erfc}\left(\frac{\eta}{\sqrt{2 \varepsilon t}}\right)\right\}\right| d \eta+\kappa \int_{0}^{t} \int_{0}^{\infty}\left|\frac{\partial}{\partial t}\left\{\operatorname{erfc}\left(\frac{\eta}{\sqrt{2 \varepsilon(t-s)}}\right)\right\}\right| d \eta d s \\
& \leq \kappa t^{-1} \int_{0}^{\infty} \frac{\eta}{\sqrt{\varepsilon t}} e^{-\eta^{2} /(4 \varepsilon t)} d \eta+\kappa \int_{0}^{t} s^{-1} \int_{0}^{\infty} \frac{\eta}{\sqrt{\varepsilon s}} e^{-\eta^{2} /(4 \varepsilon s)} d \eta d s \\
& \leq \kappa\left(t^{-\frac{1}{2}}+t^{\frac{1}{2}}\right) \varepsilon^{\frac{1}{2}}
\end{aligned}
$$

hence $(4.32)_{1}$ follows.

From (4.16) and (4.19), one can easily verify $(4.32)_{2}$, and thus the proof of Lemma 4.5 is now complete.

REMARK 4.6. Concerning all the estimates in this section, from the proofs of the lemmas, one can observe that the singularities in time $t$ are caused by the noncompatible initial data; see Remark 4.1 as well. Hence, if $\boldsymbol{u}_{0} \in V$, all the bounds associated with $t$ can be replaced by $T^{N}$ for sufficiently large $N \geq 0$.

\section{Error analysis: Proof of Theorem 2.7}

Proof. We set the corrected difference as

$$
\boldsymbol{w}:=\boldsymbol{u}^{\varepsilon}-\boldsymbol{u}^{0}-\Theta^{0}
$$

where $\boldsymbol{u}^{\varepsilon}$ and $\boldsymbol{u}^{0}$ are the Stokes and linearized Euler solutions and $\boldsymbol{\Theta}^{0}$ is the divergence-free corrector, defined in (4.2), (4.12), and (4.13), which satisfies the boundary and initial conditions appearing in (4.4) and (4.5). Then, using (1.2), (1.5), (4.4), (4.5), and (4.17), we write the equations for $\boldsymbol{w}$ :

$$
\left\{\begin{array}{rlrl}
\frac{\partial \boldsymbol{w}}{\partial t}-\varepsilon \Delta \boldsymbol{w}+\nabla\left(p^{\varepsilon}-p^{0}\right) & =\varepsilon \Delta \boldsymbol{u}^{0}+R\left(\boldsymbol{\theta}^{0}\right)+R\left(\boldsymbol{\varphi}^{0}\right)+\text { e.s.t., } & \text { in } \Omega \times(0, T), \\
\operatorname{div} \boldsymbol{w} & =0, & & \text { in } \Omega \times(0, T), \\
\boldsymbol{w} & =0, & & \text { on } \partial \Omega \times(0, T), \\
\left.\boldsymbol{w}\right|_{t=0} & =0, & & \text { in } \Omega,
\end{array}\right.
$$

where

$$
R(\boldsymbol{v}):=\frac{\partial \boldsymbol{v}}{\partial t}-\varepsilon \Delta \boldsymbol{v}
$$

for any smooth vector field $\boldsymbol{v}$ in $\Omega \times(0, T)$. 
Using (4.20) and Lemma 4.5, we see that

$$
\left\|R\left(\varphi^{0}\right)\right\|_{L^{2}(\Omega)}=\left\|R\left(\varphi^{0}\right)\right\|_{L^{2}\left(\Omega_{3 \delta}\right)} \leq \kappa\left(t^{-\frac{1}{2}}+t^{\frac{3}{2}}\right) \varepsilon^{\frac{1}{2}} .
$$

Now we multiply (5.2) by $\boldsymbol{w}$ and integrate over $\Omega$. Then, thanks to (5.4), we find $\frac{1}{2} \frac{d}{d t}\|\boldsymbol{w}\|_{L^{2}(\Omega)}^{2}+\varepsilon\|\nabla \boldsymbol{w}\|_{L^{2}(\Omega)}^{2} \leq \kappa\left(t^{-\frac{1}{2}}+t^{\frac{3}{2}}\right) \varepsilon^{\frac{1}{2}}\|\boldsymbol{w}\|_{L^{2}(\Omega)}+\left\|R\left(\boldsymbol{\theta}^{0}\right)\right\|_{L^{2}(\Omega)}\|\boldsymbol{w}\|_{L^{2}(\Omega)}$.

To estimate the term $\left\|R\left(\boldsymbol{\theta}^{0}\right)\right\|_{L^{2}(\Omega)}$, using (3.8) and (4.20), we write

$$
\begin{aligned}
\left\|R\left(\boldsymbol{\theta}^{0}\right)\right\|_{L^{2}(\Omega)} & =\left\|R\left(\boldsymbol{\theta}^{0}\right)\right\|_{L^{2}\left(\Omega_{3 \delta}\right)} \\
\leq \sum_{i=1}^{2} \| & \frac{\partial \theta^{0, i}}{\partial t}-\varepsilon \frac{\partial^{2} \theta^{0, i}}{\partial \xi_{3}^{2}}\left\|_{L^{2}\left(\Omega_{3 \delta}\right)}+\varepsilon \sum_{i=1}^{3}\right\| \mathcal{S}^{i} \boldsymbol{\theta}^{0}+\mathcal{L} \theta^{0, i} \|_{L^{2}\left(\Omega_{3 \delta}\right)} \\
& +\left\|\frac{\partial \theta^{0,3}}{\partial t}\right\|_{L^{2}\left(\Omega_{3 \delta}\right)}+\varepsilon\left\|\frac{\partial^{2} \theta^{0,3}}{\partial \xi_{3}^{2}}\right\|_{L^{2}\left(\Omega_{3 \delta}\right)} .
\end{aligned}
$$

Thanks to (4.10), (4.18), and Lemma 4.3, we find that

$$
\begin{aligned}
\sum_{i=1}^{2}\left\|\frac{\partial \theta^{0, i}}{\partial t}-\varepsilon \frac{\partial^{2} \theta^{0, i}}{\partial \xi_{3}^{2}}\right\|_{L^{2}\left(\Omega_{3 \delta}\right)} & \leq \kappa \varepsilon \sum_{i=1}^{2}\left\{\left\|\frac{\partial \bar{\theta}^{0, i}}{\partial \xi_{3}}\right\|_{L^{2}\left(\Omega_{3 \delta}\right)}+\left\|\bar{\theta}^{0, i}\right\|_{L^{2}\left(\Omega_{3 \delta}\right)}\right\} \\
& \leq \kappa\left(t^{-\frac{1}{4}}+t^{\frac{5}{4}}\right) \varepsilon^{\frac{3}{4}}
\end{aligned}
$$

Moreover, recalling that $\mathcal{S}^{i}, 1 \leq i \leq 3$, is a linear combination of the tangential differential operators and that $\mathcal{L}$ is proportional to $\partial / \partial \xi_{3}$, using (4.25) and Lemma 4.4, we see that

$$
\varepsilon \sum_{i=1}^{3}\left\|\mathcal{S}^{i} \boldsymbol{\theta}^{0}+\mathcal{L} \theta^{0, i}\right\|_{L^{2}\left(\Omega_{3 \delta}\right)}+\left\|\frac{\partial \theta^{0,3}}{\partial t}\right\|_{L^{2}\left(\Omega_{3 \delta}\right)}+\varepsilon\left\|\frac{\partial^{2} \theta^{0,3}}{\partial \xi_{3}^{2}}\right\|_{L^{2}\left(\Omega_{3 \delta}\right)} \leq \kappa\left(t^{-\frac{1}{4}}+t^{\frac{7}{4}}\right) \varepsilon^{\frac{3}{4}} .
$$

Combining (5.6)-(5.8), we obtain

$$
\left\|R\left(\boldsymbol{\theta}^{0}\right)\right\|_{L^{2}(\Omega)} \leq \kappa\left(t^{-\frac{1}{4}}+t^{\frac{7}{4}}\right) \varepsilon^{\frac{3}{4}} .
$$

From (5.5) and (5.9), we then notice that

$$
\frac{d}{d t}\|\boldsymbol{w}\|_{L^{2}(\Omega)}^{2}+\varepsilon\|\nabla \boldsymbol{w}\|_{L^{2}(\Omega)}^{2} \leq \kappa\left(t^{-\frac{1}{2}}+t^{\frac{7}{4}}\right) \varepsilon^{\frac{1}{2}}\|\boldsymbol{w}\|_{L^{2}(\Omega)} .
$$

Dropping the second term on the left hand side of (5.10) and using Young's inequality on the right hand side of (5.10), we write

$$
\frac{d}{d t}\|\boldsymbol{w}\|_{L^{2}(\Omega)}^{2}-\frac{1}{2} t^{-\frac{1}{2}}\|\boldsymbol{w}\|_{L^{2}(\Omega)}^{2} \leq \kappa\left(t^{-\frac{1}{2}}+t^{3}\right) \varepsilon
$$

Multiplying by the integrating factor $e^{-t^{1 / 2}}$, we infer from (5.11) that

$$
\frac{d}{d t}\left(e^{-t^{\frac{1}{2}}}\|\boldsymbol{w}\|_{L^{2}(\Omega)}^{2}\right) \leq \kappa\left(t^{-\frac{1}{2}}+t^{3}\right) e^{-t^{\frac{1}{2}}} \varepsilon \leq \kappa\left(t^{-\frac{1}{2}}+t^{3}\right) \varepsilon .
$$

Integrating (5.12) over $(0, t)$ for any $0<t<T$, we find

$$
\|\boldsymbol{w}\|_{L^{\infty}\left(0, T ; L^{2}(\Omega)\right)}^{2} \leq \kappa\left(1+T^{4}\right) e^{T^{\frac{1}{2}}} \varepsilon .
$$


Thanks to (4.17), (4.25), (5.1), and lemmas 4.4 and 4.5, the convergence results $(2.7)_{1}$ and (2.8) in Theorem 2.7 follow from (5.13).

To verify $(2.7)_{2}$, we come back to $(5.10)$ and integrate it over $(0, T)$. Then, using (5.13) as well, we find

$$
\|\boldsymbol{w}\|_{L^{2}(\Omega)}^{2}(T)+\varepsilon \int_{0}^{T}\|\nabla \boldsymbol{w}\|_{L^{2}(\Omega)}^{2} d t \leq \kappa\left(1+T^{\frac{19}{4}}\right) e^{T^{\frac{1}{2}} / 2} \varepsilon .
$$

Then $(2.7)_{2}$ follows from (5.14) because of (4.17), (5.1), and Lemma 4.5. The proof of Theorem 2.7 is now complete.

REMARK 5.1. Focusing on the vanishing viscosity limit result (2.8), instead of the corrector $\boldsymbol{\Theta}^{0}$ defined in Section 4 , one can use a simple corrector $\widetilde{\boldsymbol{\theta}}=\sum_{i=1}^{3} \widetilde{\theta}^{i} \boldsymbol{e}_{i}$, where

$$
\left\{\begin{array}{l}
\widetilde{\theta}^{i}(\boldsymbol{\xi} ; t):=-\left.\varepsilon^{\frac{1}{2}} \widetilde{u}^{0, i}\left(\xi^{\prime} ; t\right) \frac{h_{i}}{h}(\boldsymbol{\xi}) \frac{h}{h_{i}}\right|_{\xi_{3}=0} \frac{\partial}{\partial \xi_{3}}\left\{\sigma\left(\xi_{3}\right)\left(1-e^{-\xi_{3} / \sqrt{\varepsilon}}\right)\right\}, \quad i=1,2, \\
\widetilde{\theta}^{3}(\boldsymbol{\xi} ; t):=\varepsilon^{\frac{1}{2}} \frac{1}{h}(\boldsymbol{\xi}) \sum_{i=1}^{2} \frac{\partial}{\partial \xi_{i}}\left\{\left.\widetilde{u}^{0, i}\left(\xi^{\prime} ; t\right) \frac{h}{h_{i}}\right|_{\xi_{3}=0}\right\} \sigma\left(\xi_{3}\right)\left(1-e^{-\xi_{3} / \sqrt{\varepsilon}}\right) .
\end{array}\right.
$$

(This type of corrector is introduced in, e.g., $[25,8]$ and [7].) The divergence-free corrector $\widetilde{\boldsymbol{\theta}}$ satisfies the desired boundary condition $\left.\widetilde{\boldsymbol{\theta}}\right|_{\Gamma}=-\left.\boldsymbol{u}^{0}\right|_{\Gamma}$. Under the regularity assumptions (1.6) and (1.7), by performing the energy estimates on $\boldsymbol{u}^{\boldsymbol{\varepsilon}}-\left(\boldsymbol{u}^{0}-\widetilde{\boldsymbol{\theta}}\right)$, which are similar to those in Section 5 , one can prove the vanishing viscosity limit result (2.8). However, with this corrector $\widetilde{\boldsymbol{\theta}}$, the convergence rates of the corrected difference are getting worse by $\varepsilon^{1 / 4}$ than those in (2.7). Namely, one can obtain

$$
\left\|\boldsymbol{u}^{\varepsilon}-\left(\boldsymbol{u}^{0}+\widetilde{\boldsymbol{\theta}}\right)\right\|_{L^{\infty}\left(0, T ; L^{2}(\Omega)\right)} \leq \kappa_{T} \varepsilon^{\frac{1}{4}}, \quad\left\|\boldsymbol{u}^{\varepsilon}-\left(\boldsymbol{u}^{0}+\widetilde{\boldsymbol{\theta}}\right)\right\|_{L^{2}\left(0, T ; H^{1}(\Omega)\right)} \leq \kappa_{T} \varepsilon^{-\frac{1}{4}}
$$

for a positive constant $\kappa_{T}:=\kappa_{T}\left(T, \boldsymbol{u}_{0}, \boldsymbol{f}, \Omega, \sigma\right)$ depending on the data, but independent of $\varepsilon$.

\section{Remarks on the higher-order expansions}

Under stronger restrictions on the data than (1.6) and (1.7), following the method of constructing the corrector in [7] (or in Section 4), one can obtain higher-order expansions of the Stokes solutions. More precisely, assuming

$$
\boldsymbol{u}_{0} \in V \cap H^{6}(\Omega), \quad \boldsymbol{f} \in L^{\infty}\left(0, T ; H^{6}(\Omega)\right), \quad \Gamma \text { is of class } C^{7},
$$

one can construct an asymptotic expansion of $\left(\boldsymbol{u}^{\varepsilon}, p^{\varepsilon}\right)$ of order $\varepsilon^{1 / 2}$ in the form

$$
\boldsymbol{u}^{\varepsilon} \simeq\left(\boldsymbol{u}^{0}+\boldsymbol{\Theta}^{0}\right)+\varepsilon^{\frac{1}{2}}\left(\boldsymbol{u}^{\frac{1}{2}}+\boldsymbol{\Theta}^{\frac{1}{2}}\right), \quad p^{\varepsilon} \simeq p^{0}+\varepsilon^{\frac{1}{2}}\left(p^{\frac{1}{2}}+q^{\frac{1}{2}}\right),
$$

which we briefly explain below.

In (6.2), $\left(\boldsymbol{u}^{0}, p^{0}\right)$ is the solution of the linearized Euler equations (1.5), and $\Theta^{0}$ the corrector defined in Section 4, but for the compatible initial data: $\boldsymbol{u}_{0}=0$ on $\Gamma$. Concerning this case, the explicit expression of $\Theta^{0}$ is given by (4.17), (4.18), and (4.19) with $\widetilde{u}^{0, i}\left(\xi^{\prime} ; 0\right)$ replaced by 0 . Hence, as explained in Remark 4.6, the corrector $\Theta^{0}$ enjoys all the estimates in Section 4.1 without any singularity in $t$. That is, all the time dependencies of the estimates for $\boldsymbol{\Theta}^{0}$ can be bounded by $T^{N}>0$ for a sufficiently large $N \geq 0$. The convergence results in Theorem 2.7 are valid as well. 
Our first task to complete the asymptotic expansion (6.2) is to cancel the error of order $\varepsilon^{1 / 2}$ caused by $\partial \varphi^{0} / \partial t$ which appears in (5.4) with $\left(t^{-1 / 2}+t^{3 / 2}\right)$ replaced by $\left(1+T^{3 / 2}\right)$. For this purpose, we introduce the functions $\boldsymbol{u}^{1 / 2}$ and $p^{1 / 2}$ in $(6.2)$ as the solution of the following linearized Euler system:

$$
\begin{cases}\frac{\partial \boldsymbol{u}^{\frac{1}{2}}}{\partial t}+\nabla p^{\frac{1}{2}}=\varepsilon^{-\frac{1}{2}} \frac{\partial \varphi^{0}}{\partial t}, & \text { in } \Omega \times(0, T), \\ \operatorname{div} \boldsymbol{u}^{\frac{1}{2}}=0, & \text { in } \Omega \times(0, T), \\ \boldsymbol{u}^{\frac{1}{2}} \cdot \boldsymbol{n}=0, & \text { on } \Gamma \times(0, T), \\ \left.\boldsymbol{u}^{\frac{1}{2}}\right|_{t=0}=0, & \text { in } \Omega .\end{cases}
$$

The well-posedness of (6.3) follows from Lemma 2.1.

Adding $\varepsilon^{1 / 2} \boldsymbol{u}^{1 / 2}$ in the expansion (6.2), we have the non-zero tangential values of $\varepsilon^{1 / 2} \boldsymbol{u}^{1 / 2}$ on $\Gamma$, and this difficulty will be resolved in the next step by constructing another corrector $\boldsymbol{\Theta}^{1 / 2}$.

In (5.9), we notice that the term $R\left(\boldsymbol{\theta}^{0}\right)$ produces some error of order $\varepsilon^{3 / 4}$, which we name as

$$
\boldsymbol{E}\left(\boldsymbol{\theta}^{0}\right)=\sum_{i=1}^{3} E_{i}\left(\boldsymbol{\theta}^{0}\right) \boldsymbol{e}_{i}
$$

where

$$
\left\|E_{i}\left(\boldsymbol{\theta}^{0}\right)\right\|_{L^{\infty}\left(0, T ; L^{2}(\Omega)\right)} \leq \kappa_{T} \varepsilon^{\frac{3}{4}},
$$

for a constant $\kappa_{T}:=\kappa_{T}\left(T, \boldsymbol{u}_{0}, \boldsymbol{f}, \Omega, \sigma\right)$ depending on the data, but independent of $\varepsilon$. In order to manage the tangential error $E_{i}\left(\boldsymbol{\theta}^{0}\right), i=1,2$, and to balance the non-zero tangential values of $\varepsilon^{1 / 2} \boldsymbol{u}^{1 / 2}$ on $\Gamma$, we define $\bar{\theta}^{1 / 2, i}, i=1,2$, as a solution of the heat equation on the half plane,

$$
\begin{cases}\frac{\partial \bar{\theta}^{\frac{1}{2}, i}}{\partial t}-\varepsilon \frac{\partial^{2} \bar{\theta}^{\frac{1}{2}, i}}{\partial \xi_{3}^{2}}=\varepsilon^{-\frac{1}{2}} E_{i}\left(\boldsymbol{\theta}^{0}\right), \xi_{3}, t>0 \\ \bar{\theta}^{\frac{1}{2}, i}=-\left.\left(\boldsymbol{u}^{\frac{1}{2}} \cdot \boldsymbol{e}_{i}\right)\right|_{\xi_{3}=0}, & \xi_{3}=0, t>0, \\ \bar{\theta}^{\frac{1}{2}, i} \rightarrow 0, & \text { as } \xi_{3} \rightarrow \infty \\ \left.\bar{\theta}^{\frac{1}{2}, i}\right|_{t=0}=0, & \xi_{3}>0 .\end{cases}
$$

Then, using (3.7) and (6.5), and following the approach in [7] (or in Section 4), we define the second corrector $\Theta^{1 / 2}:=\sum_{i=1}^{3} \Theta^{1 / 2, i} \boldsymbol{e}_{i}$ where

$$
\left\{\begin{array}{l}
\Theta^{\frac{1}{2}, i}(\boldsymbol{\xi} ; t)=\left.\frac{h_{i}}{h}(\boldsymbol{\xi}) \frac{h}{h_{i}}\right|_{\xi_{3}=0} \frac{\partial}{\partial \xi_{3}}\left\{\sigma\left(\xi_{3}\right) \int_{0}^{\xi_{3}} \bar{\theta}^{\frac{1}{2}, i}\left(\xi^{\prime}, \eta ; t\right) d \eta\right\}, \quad i=1,2, \\
\Theta^{\frac{1}{2}, 3}(\boldsymbol{\xi} ; t)=-\frac{1}{h}(\boldsymbol{\xi}) \sigma\left(\xi_{3}\right) \int_{0}^{\xi_{3}} \sum_{i=1}^{2} \frac{\partial}{\partial \xi_{i}}\left\{\left.\frac{h}{h_{i}}\right|_{\xi_{3}=0} \bar{\theta}^{\frac{1}{2}, i}\left(\xi^{\prime}, \eta ; t\right)\right\} d \eta .
\end{array}\right.
$$

Under this setting, the divergence-free corrector $\Theta^{1 / 2}$ satisfies the desired boundary condition,

$$
\left.\Theta^{\frac{1}{2}}\right|_{\Gamma}=-\left.\boldsymbol{u}^{\frac{1}{2}}\right|_{\Gamma}
$$


Moreover, using the estimates on $\bar{\theta}^{1 / 2, i}, i=1,2$, which appear in, e.g., [6], one can verify that the second corrector $\Theta^{1 / 2}$ satisfies the same type of estimates as those of the first corrector $\boldsymbol{\Theta}^{0}$.

Finally, to manage the error $E_{3}\left(\boldsymbol{\theta}^{0}\right)$ in the normal direction $\boldsymbol{e}_{3}$, we define a corrector $q^{1 / 2}$ of pressures as any solution of the equation

$$
\frac{\partial q^{\frac{1}{2}}}{\partial \xi_{3}}=\varepsilon^{-\frac{1}{2}} E_{3}\left(\boldsymbol{\theta}^{0}\right)
$$

Then, by adding $\varepsilon^{1 / 2} q^{1 / 2}$ in the expansion (6.2), the term $\varepsilon^{1 / 2} \nabla q$, which appears in the error analysis, cancels the error $E_{3}\left(\boldsymbol{\theta}^{0}\right)$ in the normal direction and produces some error of order $\varepsilon^{5 / 4}$ in the tangential directions.

By performing the error analysis on the difference between $\boldsymbol{u}^{\varepsilon}$ and the proposed expansion (6.2), one can prove the following convergence result, which we state without proof: Under the regularity Assumption (6.1), we have

$$
\left\{\begin{array}{l}
\left\|\boldsymbol{u}^{\varepsilon}-\left(\boldsymbol{u}^{0}+\boldsymbol{\Theta}^{0}\right)-\varepsilon^{\frac{1}{2}}\left(\boldsymbol{u}^{\frac{1}{2}}+\boldsymbol{\Theta}^{\frac{1}{2}}\right)\right\|_{L^{\infty}\left(0, T ; L^{2}(\Omega)\right)} \leq \kappa_{T} \varepsilon, \\
\left\|\boldsymbol{u}^{\varepsilon}-\left(\boldsymbol{u}^{0}+\boldsymbol{\Theta}^{0}\right)-\varepsilon^{\frac{1}{2}}\left(\boldsymbol{u}^{\frac{1}{2}}+\boldsymbol{\Theta}^{\frac{1}{2}}\right)\right\|_{L^{2}\left(0, T ; H^{1}(\Omega)\right)} \leq \kappa_{T} \varepsilon^{\frac{1}{2}} .
\end{array}\right.
$$

Following the idea in this section, one can obtain an asymptotic expansion of the Stokes solutions at any order of $\varepsilon^{N / 2}, N \geq 0$.

Acknowledgments. The author would like to thank to James P. Kelliher, Milton Lopes Filho, and Helena Nussenzveig Lopes for suggesting the boundary layers projects concerning non-compatible initial data, and for their advice. The author also would like to thank to Makram Hamouda, Anna Mazzucato, and Roger Temam for helpful discussions, and to Nader Masmoudi for pointing out the issue on the differential operator $\mathcal{L}$ in Remark 3.1. This work was supported by NSF grant DMS 1212141 and AMS-Simons travel grants.

\section{REFERENCES}

[1] J.R. Cannon, The One-Dimensional Heat Equation, Encyclopedia of Mathematics and its Applications, Addison-Wesley Publishing Company Advanced Book Program, Reading, MA, 23, 1984.

[2] P.G. Ciarlet, An introduction to differential geometry with applications to elasticity, Journal of Elasticity, 78-79(1-3), 1-215, 2005.

[3] B. Desjardins, E. Grenier, P.L. Lions, and N. Masmoudi, Incompressible limit for solutions of the isentropic Navier-Stokes equations with Dirichlet boundary conditions, J. Math. Pures Appl., 78(5), 461-471, 1999.

[4] E. Weinan, Boundary layer theory and the zero-viscosity limit of the Navier-Stokes equation, Acta Math. Sin. (Engl. Ser.), 16(2), 207-218, 2000.

[5] G.-M. Gie, M. Hamouda, and R. Temam, Asymptotic analysis of the Stokes problem on general bounded domains: The case of a characteristic boundary, Appl. Anal., 89(1), 49-66, 2010.

[6] G.-M. Gie, M. Hamouda, and R. Temam, Boundary layers in smooth curvilinear domains: Parabolic problems, Disc. Cont. Dyn. Syst., 26(4), 1213-1240, 2010.

[7] G.-M. Gie, M. Hamouda, and R. Temam, Asymptotic analysis of the Navier-Stokes equations in a curved domain with a non-characteristic boundary, Netw. Heterog. Media, 7(4), 741-766, 2012.

[8] G.-M. Gie and J.P. Kelliher, Boundary layer analysis of the Navier-Stokes equations with generalized Navier boundary conditions, J. Diff. Equ., 253(6), 1862-1892, 2012.

[9] E. Grenier and O. Guès, Boundary layers for viscous perturbations of noncharacteristic quasilinear hyperbolic problems, J. Diff. Equ., 143(1), 110-146, 1998.

[10] M. Hamouda and R. Temam, Some singular perturbation problems related to the Navier-Stokes equations, World Sci. Publ., N.J. Hackensack, 197-227, 2007. 
[11] M. Hamouda and R. Temam, Boundary layers for the Navier-Stokes equations. The case of a characteristic boundary, Georgian Math. J., 15(3), 517-530, 2008.

[12] D. Han, A.L. Mazzucato, D. Niu, and X. Wang, Boundary layer for a class of nonlinear pipe flow, J. Diff. Equ., 252(12), 6387-6413, 2012.

[13] D. Iftimie and F. Sueur, Viscous boundary layers for the Navier-Stokes equations with the Navier slip conditions, Arch. Ration. Mech. Anal., 199(1), 145-175, 2011.

[14] W. Klingenberg, A Course in Differential Geometry, Springer-Verlag, New York, 51, 1978.

[15] M.C. Lopes Filho, A.L. Mazzucato, and H.J. Nussenzveig Lopes, Vanishing viscosity limit for incompressible flow inside a rotating circle, Phys. D, 237(10-12), 1324-1333, 2008.

[16] M.C. Lopes Filho, A.L. Mazzucato, H.J. Nussenzveig Lopes, and M. Taylor, Vanishing viscosity limits and boundary layers for circularly symmetric 2D flows, Bull. Braz. Math. Soc. (N.S.), 39(4), 471-513, 2008.

[17] N. Masmoudi, The Euler limit of the Navier-Stokes equations, and rotating fluids with boundary, Arch. Ratio. Mech. Anal., 142(4), 375-394, 1998.

[18] N. Masmoudi and F. Rousset, Uniform regularity for the Navier-Stokes equation with Navier boundary condition, Arch. Ration. Mech. Anal., 203(2), 529-575, 2012.

[19] A. Mazzucato, D. Niu, and X. Wang, Boundary layer associated with a class of $3 D$ nonlinear plane parallel channel flows, Indiana Univ. Math. J., 60(4), 1113-1136, 2011.

[20] A. Mazzucato and M. Taylor, Vanishing viscosity plane parallel channel flow and related singular perturbation problems, Anal. PDE, 1(1), 35-93, 2008.

[21] A. Mazzucato and M. Taylor, Vanishing viscosity limits for a class of circular pipe flows, Commun. Part. Diff. Equ., 36(2), 328-361, 2011.

[22] T. Nguyen and F. Sueur, Boundary-layer interactions in the plane-parallel incompressible flows, Nonlin., 25, 3327-3342, 2012.

[23] L. Prandtl, Verhandlungen des dritten internationalen mathematiker-kongresses in Heidelberg 1904, 484-491, 1905.

[24] R. Temam and X. Wang, Asymptotic analysis of Oseen type equations in a channel at small viscosity, Indiana Univ. Math. J., 45(3), 863-916, 1996.

[25] R. Temam and X. Wang, Boundary layers associated with incompressible Navier-Stokes equations: The noncharacteristic boundary case, J. Diff. Equ., 179(2), 647-686, 2002.

[26] R. Temam, Navier-Stokes Equations, AMS Chelsea Publishing, Providence, RI, 2001. Theory and numerical analysis, reprint of the 1984 Edition.

[27] R. Temam and X.M. Wang, Asymptotic analysis of the linearized Navier-Stokes equations in a channel, Diff. Integral Equ., 8(7), 1591-1618, 1995.

[28] R. Temam and X. Wang, Asymptotic analysis of the linearized Navier-Stokes equations in a general 2D domain, Asymptot. Anal., 14(4), 293-321, 1997.

[29] R. Temam and X. Wang, On the behavior of the solutions of the Navier-Stokes equations at vanishing viscosity, Ann. Scuola Norm. Sup. Pisa Cl. Sci., (4), 25(3-4), 807-828, 1997.

[30] M.I. Višik and L.A. Ljusternik, Regular degeneration and boundary layer for linear differential equations with small parameter, Amer. Math. Soc. Transl., 2(20), 239-364, 1962. 